\title{
Correction to: Mapping the knowledge of green consumption: a meta-analysis
}

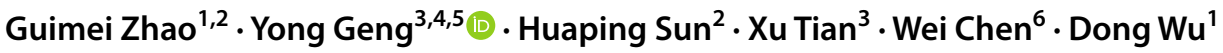 \\ Published online: 18 February 2022 \\ ○) Springer-Verlag GmbH Germany, part of Springer Nature 2022
}

\section{Correction to: Environmental Science and Pollution Research (2020) 27:44937-44950 https://doi.org/10.1007/s11356-020-11029-y}

The authors regret that the printed version of the above article contained an error for the corresponding author's affiliation. The correct and final version follows.

Correspondence to: Y. Geng, School of International and Public Affairs, Shanghai Jiao Tong University, Shanghai 200030, China

The authors would like to apologise for any inconvenience caused.

Publisher's note Springer Nature remains neutral with regard to jurisdictional claims in published maps and institutional affiliations.

The original article can be found online at https://doi.org/10.1007/ s11356-020-11029-y.

Yong Geng

ygeng@sjtu.edu.cn

1 School of Environmental Science and Engineering, Shanghai Jiao Tong University, Shanghai 200240, People's Republic of China

2 School of Finance and Economics, Jiangsu University, Zhenjiang 212013, People's Republic of China

3 School of International and Public Affairs, Shanghai Jiao Tong University, Shanghai 200030, China

4 China Institute for Urban Governance, Shanghai Jiao Tong University, Shanghai 200030, People's Republic of China

5 School of Management, China University of Mining and Technology, Xuzhou 221116, People's Republic of China

6 School of Geography and Environment, Shandong Normal University, Jinan 250358, People's Republic of China 